Supporting Information

\title{
Microfabricated Probiotic Formulation for Inhibition of Salmonella
}

\author{
Manika Chopra ${ }^{a}$, Surjith Kumar Kumaran ${ }^{a}$, Euna Oh ${ }^{a}$, Seok Chan Park ${ }^{\text {b,c }}$, Seong-O

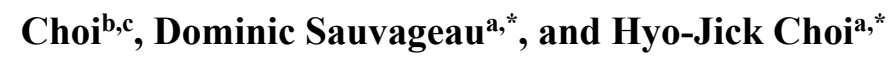

${ }^{a}$ Department of Chemical and Materials Engineering, University of Alberta, Edmonton, AB T6G 1H9, Canada

${ }^{\mathrm{b}}$ Department of Anatomy and Physiology, College of Veterinary Medicine, Kansas State University, Manhattan, KS 66506, USA

c Nanotechnology Innovation Center of Kansas State, Kansas State University, Manhattan, KS 66506, USA

*Correspondence to: dominic.sauvageau@ualberta.ca (D.S.), hyojick@ualberta.ca (H.-J.C.) 


\section{Supporting Information}

A

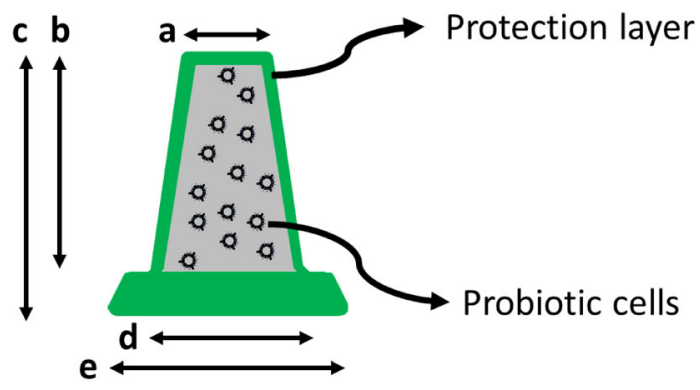
a: $380 \mu \mathrm{m}$
b: $400 \mu \mathrm{m}$
c: $550 \mu \mathrm{m}$
d: $680 \mu \mathrm{m}$
e: $760 \mu \mathrm{m}$

B

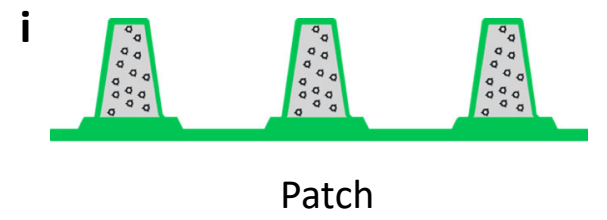

ii

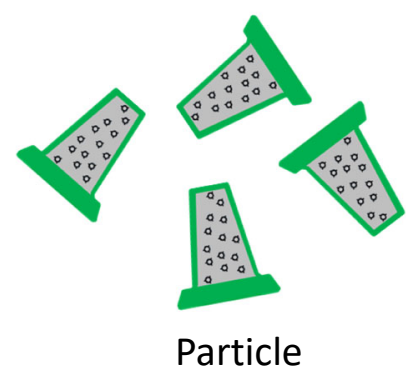

Fig. S1. Schematic representation of the microstructural architecture of the concepted conical frustum probiotics (A) fabricated via molding process in the form of patch (B-i) and particles (B-ii). 


\section{Supporting Information}
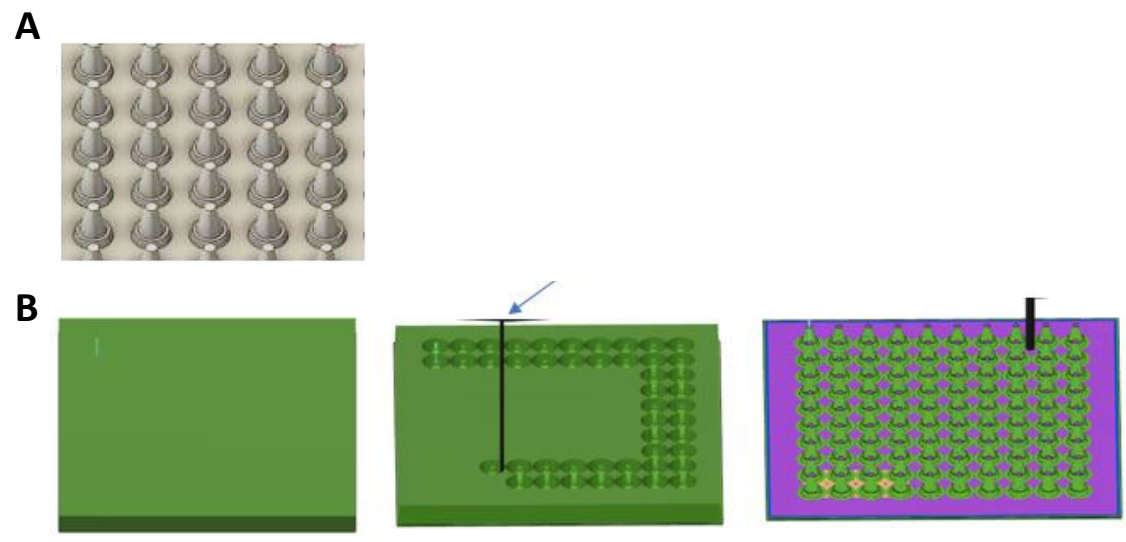

Aluminum (Al) block

Al master mold structure

C
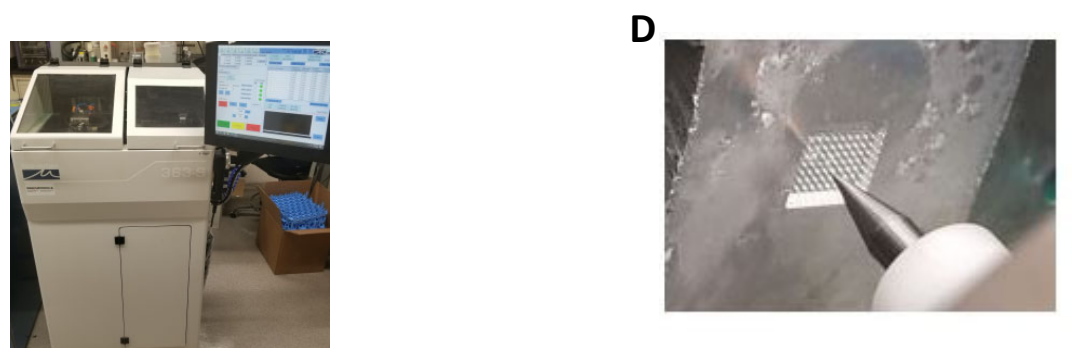

Fig. S2. Design and fabrication of Al master structure with conical-frustum architecture with a micromilling machine. (A) Computer aided design (CAD), (B) simulation, (C) CNC machine, and (D) live image of the master structure. 

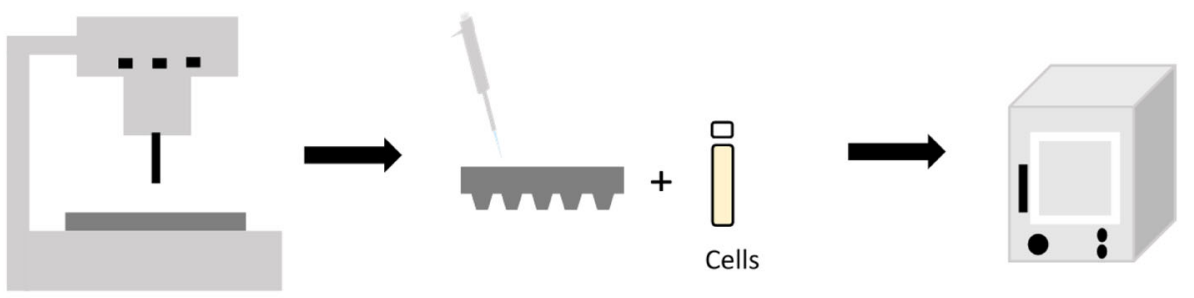

\section{Micromilling}

Encapsulation and molding
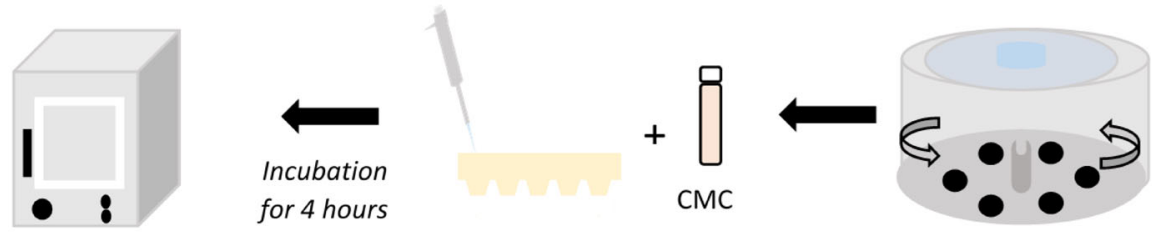

Vacuum oven

CMC backing

Centrifuge

$$
\downarrow \begin{gathered}
\text { Incubation for } \\
24 \text { hours }
\end{gathered}
$$
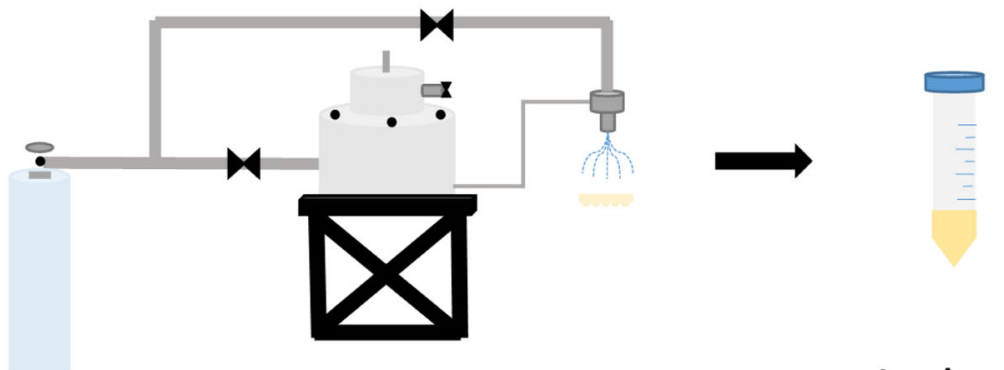

Spray coating

Incubated in MRS Broth

Fig. S3. Schematic representation of the probiotic patch fabrication process. 


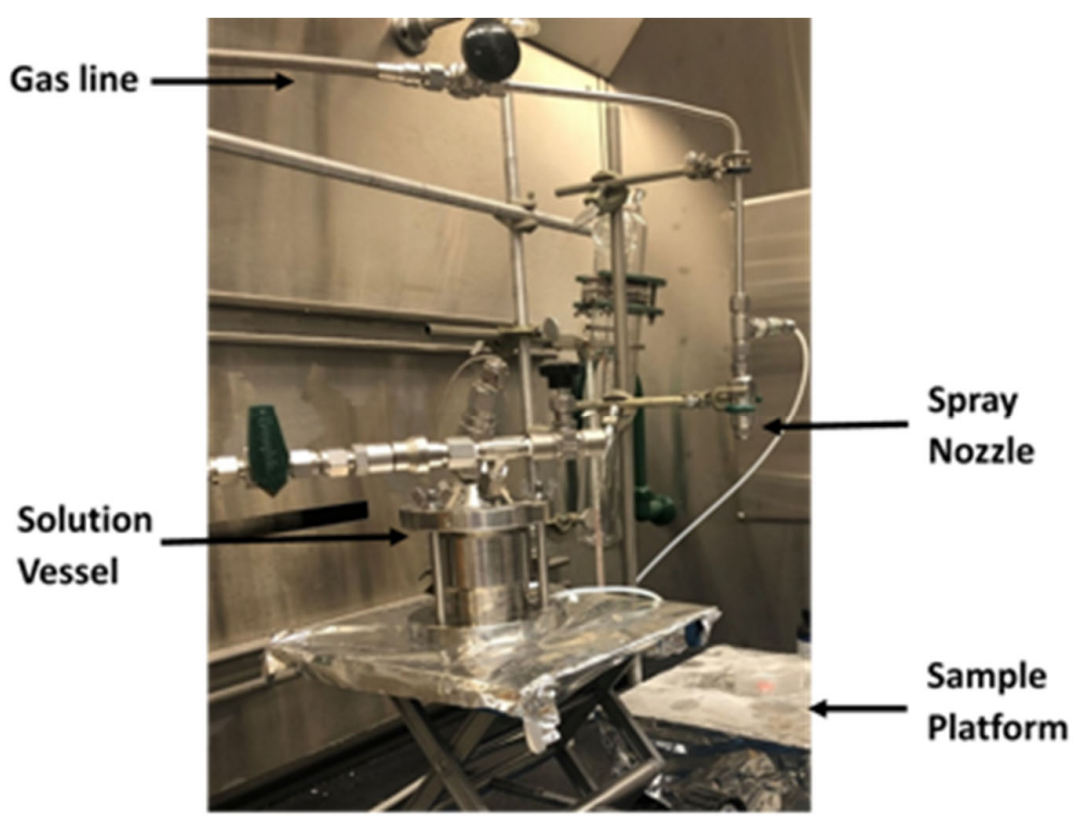

Fig. S4. Spray coating process set up. 

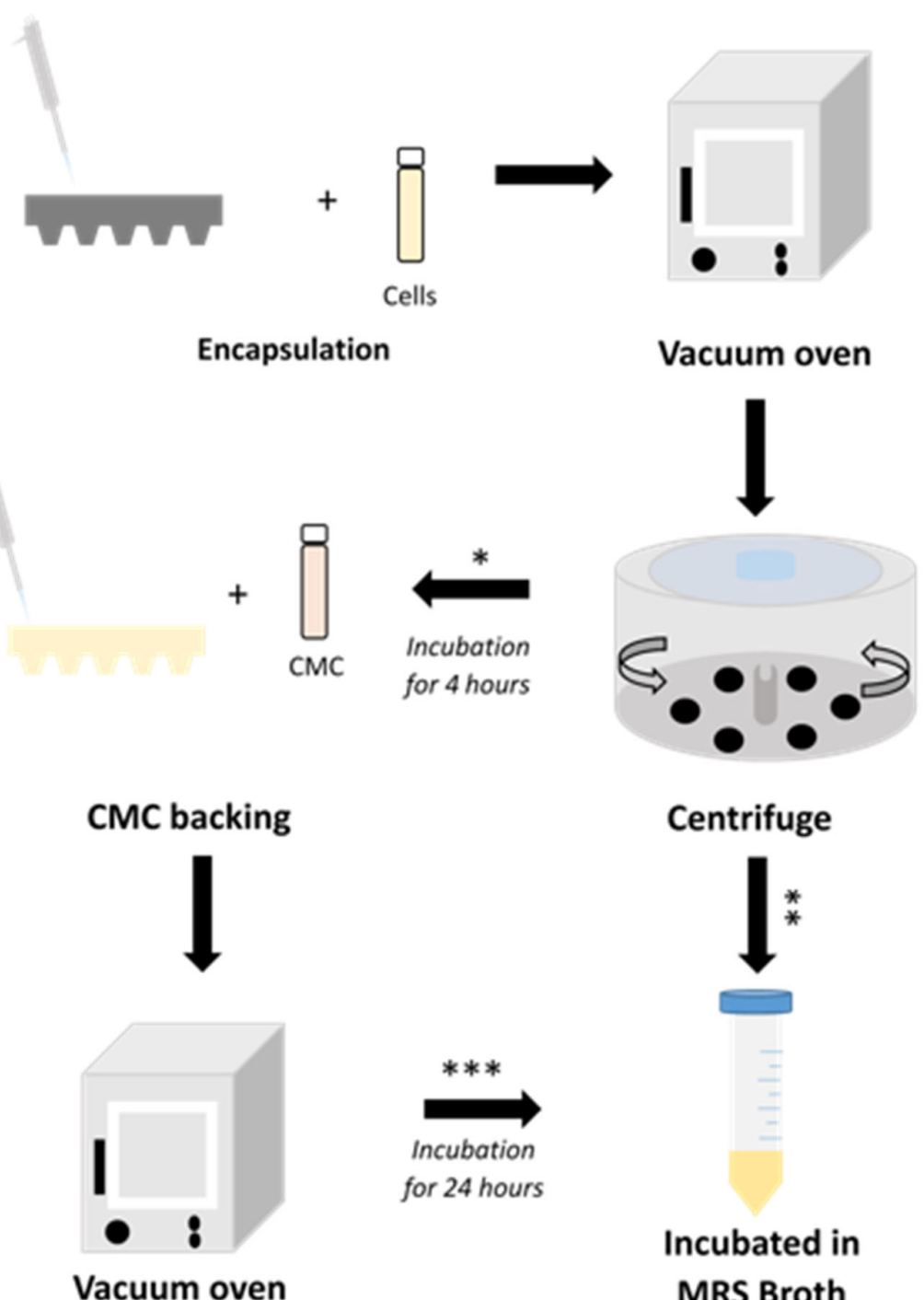

Fig. S5. Schematic representation of the probiotic patch fabrication process. Note: * Molded w/o backing, ${ }^{* *}$ Molded w/o backing MRS, ${ }^{* * *}$ Demolded w/ backing MRS. 
A

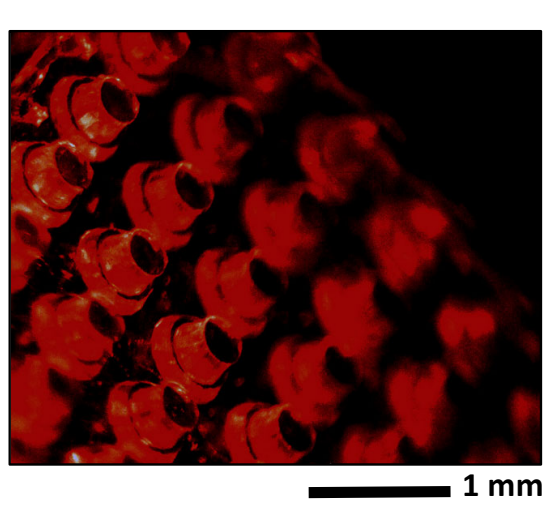

B

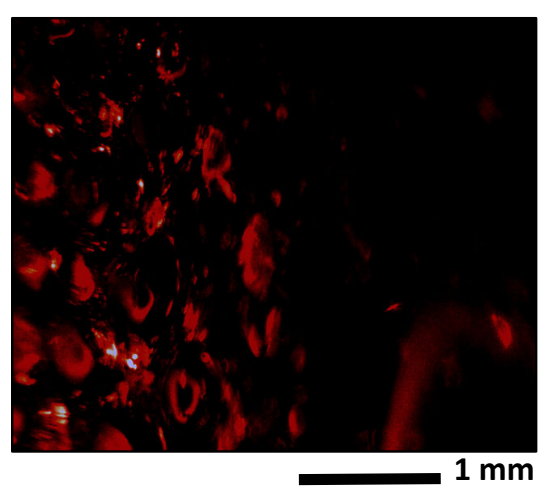

Fig. S6. Fluorescent images of SB dye encapsulated molds release behavior. (A) Before and (B) after exposure to DI for 90 seconds.

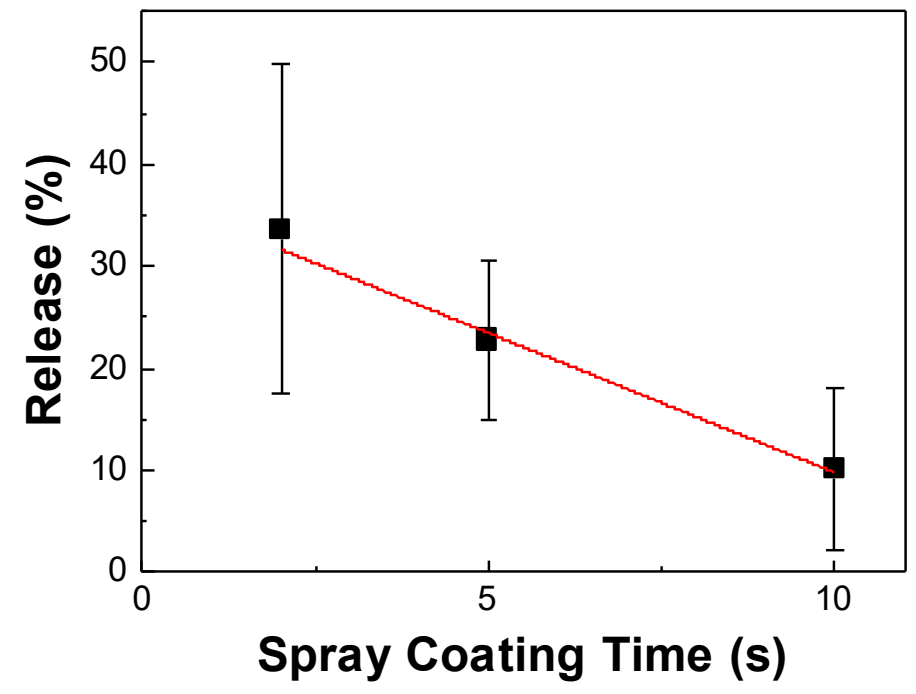

Fig. S7. Release of SB dye in simulated gastric conditions as determined by experimental release rate equations for various periods of time a sample is spray coated. 


\section{Supporting Information}
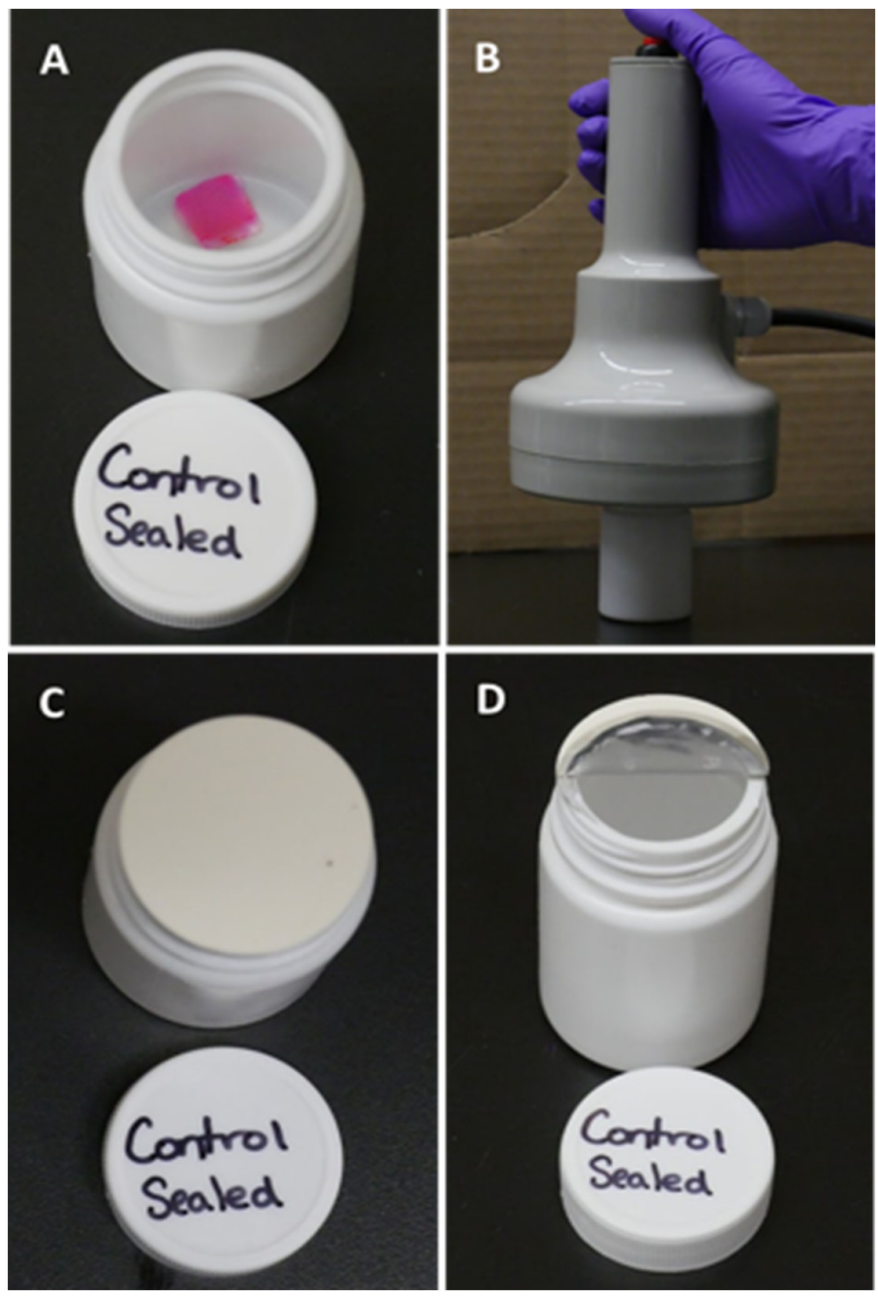

Fig. S8. Sealing of samples for stability tests. (A) Patch encapsulated with SB dye in a pill bottle, (B) sealing of bottles foil seal using electromagnetic induction sealer, (C) visual confirmation of successful sealing of foil, and (D) removal of foil seal from bottle. 